\title{
Ciência e Educação Física no Brasil: Análise das Citações Utilizadas nos Artigos das Subáreas Biodinâmica do Movimento e Sociocultural e Pedagógica Ciencia y Educación Física en Brasil: Análisis de las Citas Utilizadas en los Artículos de los Sub Campos de la Biodinámica del Movimiento y de la Sociocultural y Pedagógica
}

\author{
*Felipe Ferreira Barros Carneiro, **Amarílio Ferreira Neto, **Wagner dos Santos
}

*Instituto Federal de Educação, Ciência e Tecnologia do Espírito Santo (Brazil), **Universidade Federal do Espírito Santo (Brazil)

\begin{abstract}
Resumo. Objetiva investigar o consumo da literatura científica pelos pesquisadores da Educação Física brasileira que publicaram nos principais periódicos desse campo científico. De caráter bibliométrico o estudo analisou o tipo documental, a vida média e a obsolescência de 17013 registros listados nas referências de uma amostra de 600 artigos publicados entre os anos de 2005 e 2016, comparando as subáreas da Educação Física: Biodinâmica do Movimento e Sociocultural e Pedagógica. Conclui-se que as duas subáreas tem predominância no uso de artigos como referencial teórico, metodológico e/ou fontes, apresentando vida média da literatura de tipo clássico-efêmero e taxas de obsolescência bastante semelhantes. Além disso, aponta para um processo de equiparação entre as subáreas no que diz respeito ao consumo da literatura em razão de uma mudança na cultura científica da Subárea Sociocultural e Pedagógica impulsionada pelos critérios de avaliação científica praticados na Área 21 pela Capes.
\end{abstract}

Palavras-chave: Educação Física. Bibliometria. Citações. Vida Média. Obsolescência.

\begin{abstract}
It aims to investigate the consumption of scientific literature by Brazilian Physical Education researchers who published in the main journals in this scientific field. Bibliometric in character, the study analyzed the documental type, half-life and obsolescence of 17013 records listed in the references of a sample of 600 papers published between 2005 and 2016, comparing the sub-areas of Physical Education: Biodynamics of Movement and Sociocultural and Pedagogical . It is concluded that the two sub-areas have a predominance in the use of articles as theoretical, methodological and / or sources, presenting a half-life of the classic-ephemeral type of literature and obsolescence rates quite similar. In addition, it points to a process of equalization between the subareas with regard to the consumption of literature due to a change in the scientific culture of the Sociocultural and Pedagogical Subarea driven by the scientific evaluation criteria practiced in Area 21 by Capes.
\end{abstract}

Keywords: Physical Education. Bibliometrics. Citation. Half-life. Obsolescence.

\section{Introdução}

No atual cenário da ciência e tecnologia no Brasil, mesmo com os problemas estruturais apontados por Corder (2004), o Sistema Nacional de Pós-Graduação (SNPG) tem se configurado como uma política pública de notório desempenho no que se refere à promoção e ao desenvolvimento qualitativo de pesquisa científica e formação de massa crítica (Centro de Gestão de Estudos Estratégicos, 2010; Nascimento, 2010; Veloso, 2003).

Ancorado em agências como a Coordenação de Aperfeiçoamento de Pessoal de Nível Superior (CAPES) e o Conselho Nacional de Desenvolvimento Científico e Tecnológico (CNPq), SNPG divide a ciência em grandes áreas do conhecimento, como Ciências da Terra, Ciências Biológicas, Engenharias, Ciências da Saúde, Ciências Agrárias, Ciências Sociais Aplicadas, Ciências Humanas, Linguística, Letras e Artes e a grande área Outros. Cada uma delas subdivide-se em áreas de conhecimento, cada uma com seus comitês e critérios de avaliação que são revistos a cada interstício de quatro anos.

A área de Educação Física encontra-se estruturada na grande área da Saúde organizada na chamada Área 21, juntamente com a Fisioterapia, Fonoaudiologia e Terapia Ocupacional.

De acordo com o documento de área da CAPES, a área de Educação Física é demarcada pela multidisciplinaridade, indicando a aproximação do campo tanto com as Ciências

Fecha recepción: 24-10-19. Fecha de aceptación: 16-04-20

Felipe Ferreira Barros Carneiro

felipefbcarneiro@gmail.com
Naturais, que vem sendo tratada academicamente como subárea da Biodinâmica do Movimento (SBM), quanto com as Humanidades, que são as práticas denominadas como subárea Sociocultural e Pedagógica (SSCP).

Essa condição coloca a área de Educação Física em uma zona de fronteira entre as características expostas por Larivière, Archambault, Gingras e Vignola-Gagné (2006) no que diz respeito à aceitação de indicadores bibliométricos, na medida em que o campo reúne em seu interior práticas científicas que se aproximam tanto das Ciências Naturais, quanto das Ciências Humanas.

Para o monitoramento da atividade científica, seu impacto e resultados, foram elaborados métodos de avaliação, quantitativos e qualitativos, que possibilitam aferir os níveis de desenvolvimento alcançados por uma área de conhecimento, quais sejam: as taxas de produtividade dos pesquisadores, o potencial de crescimento das instituições e cursos e a determinação de escalas de prioridades para a distribuição de recursos.

O campo de pesquisa que se propõe aos estudos dessa natureza é conhecido como bibliometria. Consiste na aplicação de técnicas estatísticas e matemáticas para descrever aspectos da literatura e de outros meios de comunicação (Vanti, 2002). A análise bibliométrica se constitui instrumento básico no estudo dos fenômenos da comunicação científica, adquirindo importância ao utilizar um método útil para mensurar a repercussão de determinados indicadores na comunidade científica. Esse tipo de investigação tem se fortalecido como uma das maneiras de avaliar a ciência.

As análises do consumo da literatura utilizada por um campo tem sido uma das maneiras de estudar as dinâmicas 
evolutivas de uma área de conhecimento, de modo a compreender suas mudanças e transformações. Da mesma maneira, é possível conhecer a trajetória de um periódico científico quanto a sua vigência no tempo, além de possibilitar a compreensão de um conjunto de trabalhos de uma organização, universidade, departamento, ou mesmo um autor em particular.

Independentemente do caso analisado, para quantificar corretamente um fenômeno é importante definir o que será medido, que ferramentas serão utilizadas e, por fim, ajustar as medidas obtidas ao modelo matemático mais adequado.

Assim, neste estudo foram analisados os padrões de consumo da literatura científica que formam a base de sustentação teórica, metodológica e analítica na comunicação científica em periódicos dos pesquisadores da área de Educação Física.

O objetivo deste estudo foi o de analisar e comparar o padrão de consumo no que diz respeito à tipologia documental e à antiguidade das referências citadas nos artigos veiculados nos periódicos científicos especializados nos estudos da SBM e da SSCP. Além disso, buscou-se investigar se o sistema de avaliação da ciência praticado na Educação Física brasileira impacta nas práticas científicas postas em circulação no campo, no que diz respeito aos padrões de consumo da literatura científica.

Iniciativas como esta ajudam a ampliar as bases compreensivas sobre um determinado aspecto de uma área de conhecimento, conforme pode ser visto em estudos de inspiração semelhante a este como os de Cardozo, Velasco, Domíngueze Reyes (2019), Chacón-Borrego, Ubago-Jimenez, Garcia, Ruz e González (2018), Moya-Mata, Ros e Chacón (2018), Peña e Pedraz (2018), Rodríguez, Rodrígueze ÁlvarezSeoane (2018), Rodriguez et al. (2019) e Cassani et al. (2020). Esses artigos, ainda que abordem temas e objetos distintos, demonstram a relevância de investigações científicas que busquem compreender o funcionamento das estruturas que formam e conformam o campo científico.

\section{Materiais e Métodos}

Trata-se de uma pesquisa do tipo quali-quantitativa, na qual os dados foram construídos tendo como fonte periódicos científicos do campo de Educação Física. O corpus de análise foi elaborado tomando como referência os periódicos mais utilizados como alvo pelos Bolsistas de Produtividade em pesquisa do CNPq da área de Educação Física, que foram separados com base em seus alinhamentos epistemológicos entre a SBM e a SSCP. A lista de bolsistas de produtividade em pesquisa do CNPQ foi recuperada no site http://plsql1.cnpq.br/divulg/ RESULTADO_PQ 102003.curso em dezembro de 2017

As publicações desses pesquisadores foram utilizadas como critério de seleção dos periódicos por entendermos que esse grupo pertence a uma parcela da comunidade acadêmica reconhecida nacionalmente por seus pares. Parte-se do pressuposto de que suas produções circulam nos periódicos mais qualificados no campo científico.

Atualmente, existem 91 bolsistas de produtividade no campo de Educação Física. Adispersão desses pesquisadores nos níveis de bolsa encontra-se da seguinte forma: sete no nível 1A; cinco no nível 1B; cinco no nível 1C; 21 no nível 1D; 52 no nível 2; e um bolsista sênior. Desse quantitativo, a SSCP conta com 6 bolsistas (três 1C, dois 1D e um nível 2), os demais pertencem à SBM.

Buscou-se nos currículos desses pesquisadores todas as publicações realizadas em periódicos científicos no período em que concorreram aos editais e foram contemplados com a bolsa de produtividade em pesquisa na área de Educação Física. Assim, foram catalogados os 10 anos das publicações dos bolsistas nível 1 e os cinco anos dos bolsistas nível 2 conforme critérios previstos na RN-028/2015, perfazendo um total de 6437 registros de publicações recuperadas entre os anos de 2001 a 2015.

Os dados foram parametrizados a partir da média ponderada entre o número de vezes que um periódico aparece e o quantitativo de pesquisadores de produtividade presentes naquela categoria. $\mathrm{O}$ quociente desse processo foi multiplicado por um Peso atribuído a cada um dos níveis de bolsa de produtividade conforme a Tabela 1.

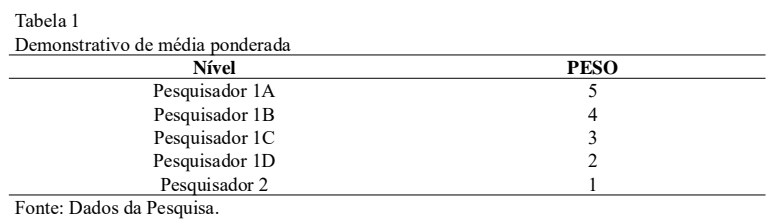

Os periódicos foram organizados em uma listagem única, onde os valores que elas receberam em cada estrato de bolsista de produtividade foram somados e ordenados de forma decrescente. Desse modo, foi possível identificar os periódicos mais relevantes para esses pesquisadores, o que pode ser considerado como representativo do campo científico, dada a contribuição e o reconhecimento desses pesquisadores na área de Educação Física.

Os periódicos selecionados (Tabela 2) contemplaram os seguintes critérios: compor o primeiro percentil do ranking de periódicos utilizados pelos bolsistas de produtividade em pesquisa e estar ranqueados entre os níveis A1 e B2 no webqualis no evento de avaliação de 2013 - 2016, indicando a relevância dos periódicos selecionados as exigências atuais do campo científico.

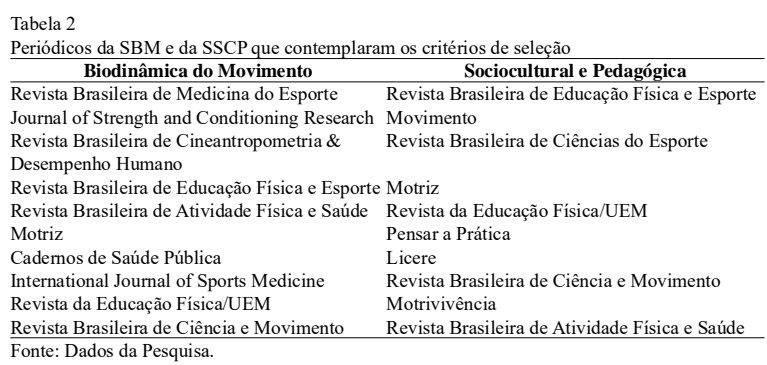

Em razão da mudança do período de avaliação da Capes de três para quatro anos no último período avaliativo encerrado em 2016, tomou-se como referência um intervalo de quatro anos, de modo que o universo de documentos foi composto por 18784 artigos publicados pelos dez periódicos da Tabela 2 entre 2005 e 2016. Os artigos foram selecionados por amostragem calculada por meio do modelo matemático de Torres, et al. (2009) utilizando os seguintes parâmetros: confiança $95 \%$, erro amostral 5\%.

A amostra foi calculada em 559 artigos, sendo 279 para 
cada subárea. Porém, uma vez que as análises foram estratificadas em três períodos (2005-2008; 2005-2012; e 2005-2016) para facilitar o planejamento, optou-se por adotar 300 artigos por subárea, totalizando 600 artigos, que foram selecionados de maneira aleatória entre os números dos periódicos e pesquisas neles veiculadas entre 2005 e 2016.

O processo de seleção aleatório tomou como base o princípio de «amostragem sistemática», definida por Torres, et al. (2009), uma vez que os periódicos mantêm uma organização interna que já dispõe de ordenamentos meticulosos.

A partir dessa delimitação utilizou-se a tabela de números aleatórios (Torres, et al., 2009) para selecionar o artigo inicial referente a cada ano dos periódicos. Após essa definição foi estabelecido um contador numérico por periódico em cada ano, tendo como referência a razão entre o total de artigos publicados naquele ano em cada periódico e o número de fascículos por eles veiculados. Assim, foram os 300 artigos da SBM (escritos por 1143 autores) e os 300 da SSCP (escritos por 851 autores)

Realizou-se uma análise sincrônica das listas de referências produzidas pelos autores dos 600 artigos que compõem a amostra. Esse tipo de análise consiste na investigação das listas bibliográficas de um conjunto de documentos de uma mesma época, de maneira a identificar o acumulado dos anos de publicação das literaturas registradas pelos pesquisadores. Esse modelo analítico foi utilizado em virtude de as listas não variarem de acordo com o tempo (Line \& Sandison, 1974).

Os dados foram compostos por 17013 registros tabulados e classificados manualmente, sendo: 9038 recuperados nos artigos da SBM e 7975 nos da SSCP.

Foram investigados os seguintes indicadores:

- Tipologia documental;

- Vida Média;

- Obsolescência;

Os indicadores foram organizados de maneira a permitir uma análise comparativa entre as duas subáreas.

\section{Resultados e Discussão}

\section{Tipologia Documental}

A tipologia documental tomou como base todos os registros listados nos artigos analisados, de maneira que a classificação considerasse as bibliografias consultadas, bem como os documentos que foram utilizados como fontes pelos autores em seus estudos.

Nesses termos, foram identificados os seguintes tipos documentais: artigos, livros, capítulos de livros, monografias, dissertações e teses, anais e resumos de eventos científicos, legislações, jornais, relatórios (científicos e técnicos), informações da web (páginas da internet, tais como blogs, redes sociais, sites especializados e informações de instituições oficiais) e outros documentos (tipos documentais que apareceram somente uma vez). Houve também um pequeno quantitativo de referências as quais não foram possíveis de classificar em razão da falta de informações nas listas bibliográficas de alguns artigos.

Job (2006), ao investigar as listas bibliográficas presentes em teses defendidas em um Programa de Pós-graduação em Educação Física, já havia identificado a predominância da citação de artigos. Porém, a autora não realizou a distinção por subáreas.

Assim, a Figura 1 apresenta a distribuição percentual dos tipos de documentos identificados nas listas de referências presentes nos 300 artigos da SSCP que foram utilizados nas análises.
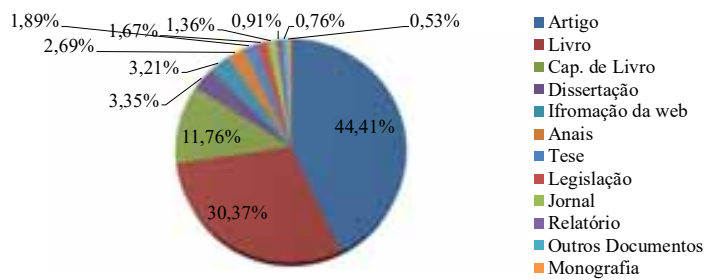

Figura 1 - Tipologia documental presente na lista de referências dos artigos da SSCP Fonte: Dados da Pesquisa

Os dados da Figura 1 revelam que, entre 2005 e 2016, nos estudos socioculturais e pedagógicos da área de Educação Física que circularam nos periódicos científicos mais relevantes, houve uma prevalência no uso de três tipos de documentos nas listas bibliográficas utilizadas pelos autores: artigos, livros e capítulo de livros.

O uso de artigos como suporte teórico e científico ocupa o maior percentual das referências utilizadas pelos autores da SSCP, com 3542 registros (44,41\%), indicando que as pesquisas publicadas nesse formato têm se configurado como as mais relevantes para os pesquisadores dessa subárea.

Os livros, com 2422 incidências (30,37\%), aparecem como o segundo tipo documental mais listado nas referências dos artigos da SSCP. Essa tendência já descrita por Job (2006) é um contraponto aos argumentos de que esse tipo de documento é mais comum nos estudos socioculturais que os artigos (Carvalho \& Manoel, 2007; Carvalho et al., 2008).

Esse cenário não se modifica nem se forem somados a esse quantitativo os 938 registros $(11,76 \%)$ de capítulos de livros que foram utilizados pelos autores dessa subárea. Não obstante, ainda que essas tipologias documentais não sejam as mais utilizadas, elas representam um percentual elevado das fontes e bibliografias utilizadas nesses estudos, sendo importante considerá-las nos processos de avaliação da ciência nessa área.

As outras categorias de documentos listados nas referências da SSCP corresponderam a 13,46\% do total de citações identificadas, o que equivale a 1073 registros utilizados no período analisado.

No caso da SBM (Figura 2), o panorama apresentado no uso de tipos documentais nas listas bibliográficas configurouse de maneira bastante distinta. Nessa subárea, observa-se a predileção dos pesquisadores por utilizar informações recolhidas de artigos científicos como elemento de sustentação argumentativa dos trabalhos, uma vez que $86,21 \%$ (7791) dos documentos registrados pertencem a essa categoria.

Esses achados se aproximam do perfil identificado por Noronha (1998) e Saes (2000) em estudos sobre Saúde Pública, e por Carvalho (2005) e Carvalho et al. (2007) na área de Saúde Coletiva, indicando a aproximação do perfil da SBM ao tipo de práticas científicas desenvolvidas nestes subcampos da área da Saúde no Brasil. 


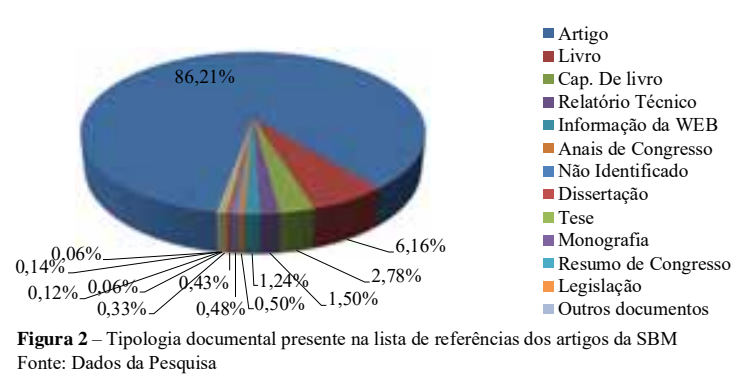

Os demais tipos documentais utilizados na SBM somaram 1247 registros nas listas bibliográficas, o que corresponde a $13,79 \%$ do total de documentos. Desse percentual, os livros e capítulos de livros representam $64,79 \%$, sendo utilizados como referência 808 vezes pelos autores dos trabalhos investigados. Os demais tipos corresponderam a 439 registros dispersos nos 300 trabalhos dessa subárea.

Quando se observa a série histórica nos períodos de 2005-2008, 2009-2012 e 2013-2016 (Tabela 3), fica evidente que o artigo é a tipologia documental mais consolidada como referência nos trabalhos produzidos sob a ótica da SBM, pois, em todos os períodos, esse tipo de documento compôs mais de $80 \%$ das listas bibliográficas disponibilizadas pelos autores dos textos analisados.

No período de 2005-2008, das 2814 referências registradas, os artigos representaram 80,35\% (2261); os livros e capítulos de livros, juntos, foram utilizados 388 vezes, o que corresponde a $13,79 \%$ dos registros. Os demais tipos documentais somaram 5,86\% (165) das citações recebidas

Entre 2009 e 2012 ocorreu um crescimento de 6,9\% no uso de artigos nas referências citadas nos trabalhos, reunindo $2519(87,25 \%)$ do total de 2887 bibliografias. Já os livros e capítulos de livros apresentaram uma tendência de queda de seis pontos percentuais, passando a compor 7,79\% (225) da literatura utilizada pelos pesquisadores da SBM. Os demais documentos, como literatura cinzenta, anais de eventos e informações da web também apresentaram redução, passando a compor 4,96\% (143) da tipologia documental citada nesse período.

No período de 2013-2016, do universo de 3173 registros bibliográficos listados nos 300 trabalhos da SBM, os artigos passaram a compor $90,45 \%$ do total de documentos, mantendo a tendência de crescimento do quadriênio anterior. Nessa mesma toada, os demais tipos de literatura mantiveram a perspectiva de queda em sua utilização, na qual livros e capítulos de livros passaram a compor 5,7\%, com 181 registros, sendo a maior baixa no percentual de livros (queda de 1,89\%). Os demais documentos tiveram pouca variação, com uma que-

\begin{tabular}{|c|c|c|c|c|c|c|}
\hline \multirow{2}{*}{ Tipo de Documento } & \multicolumn{3}{|c|}{ SBM } & \multicolumn{3}{|c|}{ SSCP } \\
\hline & $2005-2008$ & $2009-2012$ & 2013-2016 & 2005-2008 & 2009-2012 & 2013-2016 \\
\hline Artigo & $80,35 \%$ & $87,25 \%$ & $90,45 \%$ & $37,39 \%$ & $42,44 \%$ & $48,97 \%$ \\
\hline Livro & $8,81 \%$ & $5,92 \%$ & $4,03 \%$ & $32,75 \%$ & $30,67 \%$ & $25,42 \%$ \\
\hline Cap. de Livro & $4,98 \%$ & $1,87 \%$ & $1,67 \%$ & $13,86 \%$ & $11,71 \%$ & $9,00 \%$ \\
\hline Dissertação & $0,89 \%$ & $0,89 \%$ & $0 \%$ & $2,80 \%$ & $4,15 \%$ & $2,66 \%$ \\
\hline Informação da web & $0,89 \%$ & $1,42 \%$ & $1,39 \%$ & $2,67 \%$ & $2,60 \%$ & $4,09 \%$ \\
\hline Anais & $0,96 \%$ & $0,42 \%$ & $0,16 \%$ & $4,00 \%$ & $2,40 \%$ & $1,65 \%$ \\
\hline Tese & $0,57 \%$ & $0,28 \%$ & $0,25 \%$ & $2,11 \%$ & $1,72 \%$ & $1,72 \%$ \\
\hline Legislação & $0,14 \%$ & $0,10 \%$ & $0,10 \%$ & $1,68 \%$ & $1,45 \%$ & $1,76 \%$ \\
\hline Jornal & $0 \%$ & $0 \%$ & $0 \%$ & $0,52 \%$ & $1,35 \%$ & $1,99 \%$ \\
\hline Relatório & $0 \%$ & $0 \%$ & $0 \%$ & $0,77 \%$ & $0,51 \%$ & $1,39 \%$ \\
\hline Outros Documentos & $0,21 \%$ & $0,03 \%$ & $0,03 \%$ & $0,82 \%$ & $0,30 \%$ & $1,16 \%$ \\
\hline Monografia & $0 \%$ & $0,03 \%$ & $0,06 \%$ & $0,65 \%$ & $0,71 \%$ & $0,19 \%$ \\
\hline Relatório Técnico & $1,67 \%$ & $1,63 \%$ & $1,23 \%$ & $0 \%$ & $0 \%$ & $0 \%$ \\
\hline $\begin{array}{l}\text { Não Identificado } \\
\text { Resumo de }\end{array}$ & $0,32 \%$ & $0,87 \%$ & $0,28 \%$ & $0 \%$ & $0 \%$ & $0 \%$ \\
\hline Congresso & $0,14 \%$ & $0 \%$ & $0 \%$ & $0 \%$ & $0 \%$ & $0 \%$ \\
\hline
\end{tabular}

da de $0,91 \%$, compondo $4,05 \%$ (122) das listas bibliográficas das pesquisas analisadas.

Os dados referentes à SBM indicam que o uso de livros, capítulos de livros, literatura cinzenta, anais de eventos e outros tipos de documentos está em processo de «extinção» na cultura científica pelos investigadores que produzem conhecimento nessa subárea.

Na SSCP o perfil dos documentos utilizados é mais diversificado do que na SBM, na medida em que se observa a presença de um percentual maior de tipos distintos de referências nos três períodos analisados. Porém, é possível inferir que, gradativamente, o perfil das listas bibliográficas dos artigos da SSCP tem se modificado.

No período de 2005 a 2008, das 2324 referências recuperadas nos trabalhos dessa subárea, os artigos representavam menos da metade dos tipos de documentos utilizados para dar suporte científico às pesquisas, compondo 37,39\% (869) das bibliografias. Os livros (761) e os capítulos de livros (322) somados representavam os documentos mais relevantes para essa comunidade científica, com $46,61 \%$ de utilização. Em conjunto, os demais tipos documentais também apareciam com um percentual expressivo, quando comparado com o perfil identificado na SBM, qual seja: $16 \%$ (372).

Porém, entre os anos de 2009 e 2012 esse panorama sofreu algumas alterações. O percentual referente ao quantitativo de artigos listados nas referências dos trabalhos recebeu um incremento de $5,05 \%$ em relação ao período anterior, atingindo a marca de 1258 registros $(42,44 \%)$, de um universo de 2964 no período.

Esse quantitativo praticamente se iguala ao do uso de livros e capítulos de livros que, somados, reúnem os valores absolutos de 1256 registros (42,38\%). Cabe salientar que esse tipo de documento apresentou uma queda de $4,23 \%$ no uso. Os demais tipos de documentos também apresentaram uma redução percentual de utilização, passando a compor $15,18 \%$ (450) dos registros bibliográficos recrutados pelos autores da SSCP.

Nos 2667 registros recuperados nos trabalhos referentes ao período de 2013-2016, as tendências sinalizadas nos anos anteriores permanecem. Os estudos publicados no formato de artigos passaram a compor quase a metade das bibliografias $(48,97 \%)$ utilizadas pelos pesquisadores da SSCP, correspondendo a 1306 documentos e um crescimento de $6,53 \%$.

O uso de livros e capítulos de livros como referência cai para $34,42 \%$ (918), assim como os outros tipos documentais, que têm o seu uso reduzido para 8,36\% (443). Esse panorama indica que, de maneira sistemática, a cultura científica, no que se refere ao uso de bibliografias de natureza diversificadas, forma no horizonte a imagem de que as características dos tipos de documentos presentes nas listas bibliográficas dos pesquisadores da SSCP tende a se tornar cada vez mais semelhante aos encontrados nas pesquisas da SBM.

É importante salientar que a partir do ano de 2006 o comitê científico da Área 21 passou a atribuir um valor maior à publicação de artigos em periódicos, em especial, naqueles indexados em bases de dados internacionais.

Souza (2018) demonstra que a política científica adotada por um país é capaz de alterar significativamente o perfil e o 
comportamento dos pesquisadores envolvidos no sistema científico. Rivero, Santos e Trzesniak (2019) corroboram essa ideia ao indicarem que as políticas de avaliação da ciência nacional utilizadas pela CAPES e CNPq são um dos fatores que influenciam as mudanças nas «práticas de publicação» das comunidades científicas. Nesse sentido, as argumentações desses autores permitem inferir que a alteração da política de avaliação ocorrida em 2006 pode ter influenciado no tipo de literatura consumida pelos autores no processo de elaboração de suas pesquisas.

Esse panorama leva a alguns apontamentos no que diz respeito aos critérios de avaliação adotados pela área. O primeiro deles considera os argumentos de Carvalho e Manoel (2007, p. 1) de que «[. . . ] a universidade é o espaço do livro por excelência» e que corresponde a uma «[. . . ] parte significativa da produção intelectual da pós-graduação» Carvalho et al. (2008, p. 1), especialmente nas áreas de humanidades, onde a SSCP guardaria relação e similaridade no que diz respeito ao formato documental privilegiado para escoamento da produção científica.

Nesse sentido, a redução do consumo de literatura no formato de livro no processo de elaboração das pesquisas na área de Educação Física pode estar relacionada às políticas científicas que primam pela publicação em periódicos, preferencialmente de impacto internacional, fazendo com que as pesquisas da SSCP que poderiam ser publicadas como livros ou capítulos de livros passassem a circular no formato de artigo, reduzindo, assim, a disponibilidade e o uso desses materiais nas listas bibliográficas da produção científica dessa subárea, o que poderia ser considerado um processo de mudança de cultura científica e/ou uma distorção gerada pelos critérios de avaliação praticados no campo.

O segundo apontamento se baseia na relevância do papel do livro para as universidades e a pós-graduação (Carvalho \& Manoel, 2007; Carvalho et al., 2008). De acordo com as informações relativas ao Qualis Livro (sistema utilizado pela Capes para avaliar livros) disponibilizado no relatório de avaliação quadrienal do período 2013-2016(CAPES, 2017), na área de Educação Física foram avaliados 465 produtos, apresentando, segundo o mesmo documento, um crescimento em relação ao período avaliativo anterior.

Desse modo, o que se observa é um crescimento na produção e avaliação de livros ao mesmo tempo em que se reduz o percentual desse tipo de documento nas listas bibliográficas dos pesquisadores da área. Nesse sentido, se compreendermos que os critérios de avaliação utilizados têm modificado a cultura científica do campo, torna-se pujante a necessidade de se reestruturarem os indicadores avaliativos, de maneira a potencializar o aproveitamento desse tipo de produção.

Outrossim, se a tendência sinalizada na Tabela 3 se mantiver, em alguns anos, o uso de livros como elementos de sustentação argumentativa das práticas científicas da SSCP que são publicadas no formato de artigo se equiparará ao contexto expresso na SBM.

Esse cenário pode indicar uma drástica mudança na cultura científica da SSCP ou que, diferentemente do que se pensava, talvez, as práticas científicas dessa subárea não sejam tão semelhantes àquelas comumente atribuídas às Ciências Humanas e Sociais. Nesse sentido, a SSCP operaria num «entre lugar» de tradições científicas distintas (?).

\section{Vida Média e Obsolescência}

A obsolescência trata da perda gradual da vigência da informação. De acordo com Line e Sandison (1974) esse fenômeno trata da perda de utilidade, do uso ou da validade dos argumentos com o passar do tempo.

Price (1986), ao estudar em distintas áreas a distribuição das referências bibliográficas por ano, identificou que a curva de envelhecimento da literatura científica é exponencial (assim como o desenvolvimento da ciência).

De acordo com Price (1986), a ciência se organiza em três fases de desenvolvimento. A primeira consiste no movimento dos «pioneiros», que são os primeiros pesquisadores a se dedicarem a investigar uma nova área, objeto ou tema científico. A segunda é a do «crescimento exponencial», na qual o desenvolvimento ocorre como um todo, ou seja, aumenta-se o número de cientistas, laboratórios, instituições, financiamento e, consequentemente, o número de artigos publicados. Por fim, atinge-se a terceira fase, do «crescimento linear», onde é possível identificar um ritmo de desenvolvimento frequente e com poucas oscilações no número de documentos produzidos. Segundo Price (1986), esse processo faz com que a ciência dobre o seu tamanho, num período entre 10 e 15 anos. De acordo com o autor, ao mesmo tempo em que o número de publicações dobra nesse período, o número de citações recebidas pelos trabalhos se divide por dois a cada treze anos e meio.

Para analisar a literatura listada nos documentos da SBM e SSCP, foi utilizado o modelo teórico de Brookes (1970), que estabeleceu uma lei matemática que descreve a perda temporal de utilidade de um conjunto de documentos, desenvolvendo um modelo matemático que tornou esse processo inteligível.

A vida média (h) é a idade em que a literatura encontra o seu ponto médio de diminuição no número de vezes que é utilizada, ou seja, é reduzida pela metade (50\%). Pode ser obtida por meio do cálculo da mediana $(\mathrm{Md})$ por interpolação linear. Para isso foi elaborada uma tabela contendo as seguintes colunas:

- ano de publicação;

- frequência absoluta $(f i)$ - número de citações recebidas no ano;

- frequência acumulada absoluta (Fia) - acumulado das citações em ordem cronológica inversa;

- frequência acumulada relativa (Fir) - valores percentuais acumulados das citações;

- idade da publicação - começando pelo zero (0) para o ano mais recente e os demais valores em ordem crescente até atingir a idade correspondente ao último ano obtido na publicação.

Após essa organização, calculou-se a mediana por meio da interpolação linear, utilizando a seguinte fórmula:

$$
M d=l+\frac{\left(\frac{n}{2}\right)-\text { Fant }}{f i} *(L-l)
$$

onde:

$=$ frequência absoluta

$=$ tamanho da amostra 
= posição da mediana

$=$ limite inferior da mediana

$=$ limite superior da mediana

= frequência absoluta acumulada, anterior à classe mediana

Calculada a vida média foi realizado o cálculo da obsolescência e o fator de envelhecimento da literatura. Esse procedimento foi realizado a partir da seguinte fórmula:

$$
a=(0,5)^{1 / h}
$$

Onde:

$=$ taxa de envelhecimento

= vida média

Conforme o modelo teórico de Brookes (1970), o resultado dessa equação é expresso em valores entre um (literatura com maior utilidade e com fator de envelhecimento reduzido) e zero (literatura com maior fator de envelhecimento).

No caso da literatura estudada, optou-se por realizar o cálculo somente dos documentos citáveis, quais sejam: artigos, livros e capítulos de livros. Porém, no caso dos livros, foi identificado um conjunto de documentos que apresentavam anos de edição e publicação distintos. Nesses casos, optou-se por normalizá-los, tomando como referência o ano mais antigo da obra citada quando as edições não apresentavam diferenças significativas entre elas, conforme as recomendações de Maroldi, Lima e Hayashi (2018). Porém, quando identificadas alterações significativas nas obras (reestruturação/inserção de capítulos ou prefácios), foram considerados os anos de publicação em que as modificações ocorreram.

Nesses termos, foi analisado um total de 6902 referências listadas nas pesquisas da SSCP (3542 artigos e 3360 livros e capítulos) e 8599 na SBM (7791 artigos e 808 livros e capítu$\operatorname{los})$.

Vida Média da Literatura nas Subáreas Biodinâmica do Movimento e Sociocultural e Pedagógica

Ao estudar a vida média da literatura utilizada em teses e dissertações da Educação Física, Job (2006) identificou que os documentos utilizados na área começam a perder a sua utilidade em torno dos 5-6 anos a partir de sua publicação. Cabe ressaltar que o estudo de Job (2006) considerou todos os tipos documentais em sua análise, e não apresentou uma normatização em relação às datas de publicação dos livros.

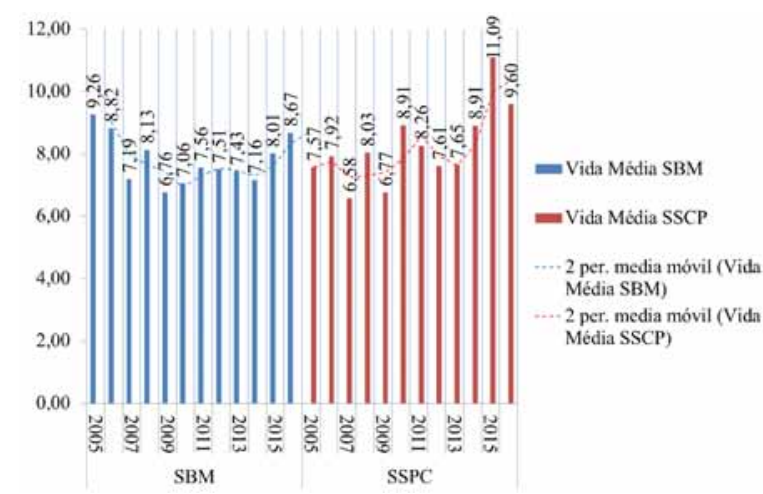

Figura 3 - Comparativo entre índices de Vida Média das referências listadas nos artigos da SBM e SSCP entre 2005 e 2016

Fonte: Dados da Pesquisa
Além disso, como já salientado neste estudo, o artigo de Job (2006) abordou os documentos da Educação Física sem separá-los em subáreas.

Quando feitas essas distinções, os dados da área se apresentam conforme a Figura a seguir.

A Figura 3 apresenta uma comparação entre as vidas médias identificadas na literatura utilizada como referência pelos autores dos 300 artigos da SBM e da SSCP, selecionados nos principais periódicos da área de Educação Física entre os anos de 2005 a 2016.

Os dados indicam que a vida média da literatura oscilou nas duas subáreas, tendo como média mais alta o valor de 9,26 anos na SBM e de 11,09 anos na SSCP. Já os valores mais baixos foram, respectivamente, de 6,77 e de 6,58.

$\mathrm{Na}$ SBM, o comportamento de citar literaturas mais antigas tem seu ápice no primeiro ano da série histórica e seu maior ponto de declínio no ano de 2009. Os demais pontos da série mantêm uma vida média com valores relativamente próximos, variando, aproximadamente, 2,5 anos no período analisado. Essa estabilidade, muito provavelmente, deve-se à prevalência no consumo de artigos como tipos de documentos privilegiados pelos autores dessa subárea em suas práticas científicas em todo o período analisado.

A SSCP tem no início de sua série histórica (2007) a média mais baixa do consumo da literatura, apresentando algumas oscilações com o passar dos anos, porém com uma leve tendência de elevação da vida média. $\mathrm{O}$ uso de literatura mais antiga foi observado no comportamento dos pesquisadores no ano de 2015, com uma nova queda no ano de 2016, apresentando uma variação maior que a SBM: 4,5 anos, aproximadamente. É interessante que a ampliação do uso de artigos em detrimento de livros e capítulos por essa comunidade de pesquisadores, aparentemente, tenha pouco efeito sobre a vida média da literatura utilizada na SSCP.

A Figura 4 demonstra a vida média das duas subáreas calculadas por período. Essa organização dos dados permite visualizar as informações em um determinado momento histórico do campo, possibilitando identificar as mudanças e/ ou manutenções de tendências na velocidade em que uma informação começa a perder a utilidade na SBM e na SSCP, durante os intervalos temporais de 2005-2008, 2009-2012 e 2013-2016.

Quando distribuídos por período, os índices de vida média da SBM apresentam uma curva em «U», na qual o valor mais elevado encontra-se no intervalo de 2005-2008, com 8,3 anos; o ponto mais baixo no período de 2009-2012, com vida média de 7,2 anos; com nova elevação no intervalo que finaliza a série histórica (2013-2016), com 7,8 anos.

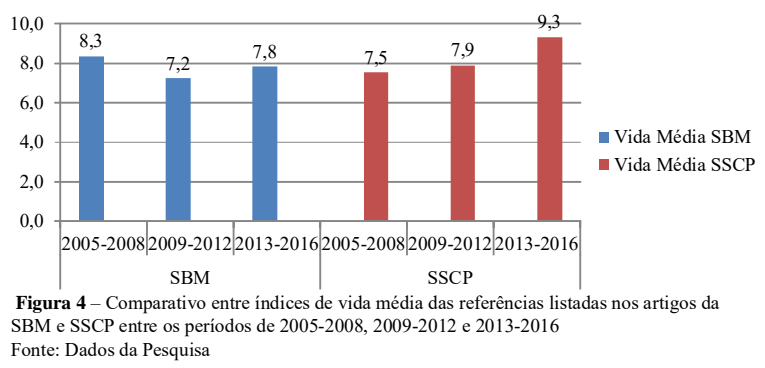

No caso da SSCP, observa-se um padrão de crescimento linear, pois a vida média recebeu um incremento de 0,4 anos entre os períodos de 2005-2008 (7,5) e 2009-2012 (7,9), e de 
1,4 anos entre 2009-2012 e 2013-2016.

Burton e Kleber (1960), ao investigarem a vida média de nove áreas do conhecimento, identificaram que o consumo da literatura variava de acordo com as características de cada campo científico, podendo ser classificadas entre Clássicas e Efêmeras. As literaturas Efêmeras são características em áreas nas quais se observa uma rápida mudança e/ou avanço de conteúdos e técnicas que fazem com que as anteriores sejam invalidadas ou inutilizadas. Já as literaturas Clássicas são comuns em campos científicos nos quais as teorizações e conceitos são elementos constantes no processo de desenvolvimento do conhecimento.

As comparações entre áreas realizadas no estudo de Burton e Kleber (1960) permitem inferir que o consumo Efêmero é demarcado por uma vida média da literatura inferior a 7 anos, enquanto a literatura clássica seria aquela com a vida média superior a 10 anos. No interstício entre 7 e 10 anos de vida média, estariam aquelas áreas com o padrão de consumo que tem sido denominado Clássico-Efêmero (Carvalho, 1975) ou Intermediário (Araújo, 2006).

Tomando como referência a classificação de Burton e Kleber (1960) e considerando as vidas médias identificadas nas listas bibliográficas utilizadas nas pesquisas da SBM e da SSCP nos períodos analisados, observa-se que o padrão de consumo da literatura científica nas duas subáreas pode ser classificado como clássico-efêmero ou intermediário.

Os dados relativos à SBM eram esperados, pois, na literatura especializada do campo, sempre houve o entendimento de que os fazeres científicos dessa subárea se aproximam daqueles praticados nas áreas biomédicas (Amadio \& Barbanti, 2000; Betti, Carvalho, Daolio \& Pires, 2004; Bracht, 2007; Carneiro, Ferreira Neto \& Santos, 2015; Kokubun, 2003; Manoel \& Carvalho, 2011).

Porém, se considerarmos que os estudos epistemológicos na área de Educação Física como os de Bracht (1993; 2006), Carneiro Ferreira Neto \& Santos (2015), Daolio (2007), Festeinseifer (2001), Manoel e Carvalho (2011), têm considerado que as pesquisas sociopedagógicas da área têm suas práticas científicas fundamentadas nas Humanidades, os achados identificados nos artigos da SSCP analisados são reveladores, uma vez que, nesse aspecto, indicam forte similaridade com o modo de fazer ciência da SBM.

Da Obsolescência da Literatura nas Subáreas Biodinâmica do Movimento e Sociocultural e Pedagógica

A obsolescência é calculada tomando como referência a vida média de um determinado conjunto de documentos. Enquanto o cálculo da vida média indica o ponto de inflexão onde a informação «perde» $50 \%$ de sua utilidade, a resultante da obsolescência representa o processo de perda gradativa da utilidade da literatura científica, de maneira que, quanto menor a vida média, maior a taxa de envelhecimento da bibliografia.

Segundo o modelo matemático de Brookes (1970), o comportamento da obsolescência pode ser representado em função de uma curva logarítmica que descende gradativamente entre os valores de 1 (mais útil) a 0 (literatura obsoleta). Os resultados desses cálculos aplicados à SBM e a SSCP encontram-se expressos nas Figuras 6 e 7.

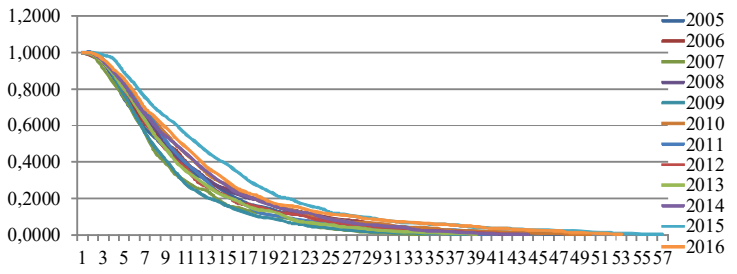

Figura 5 - Padrão de obsolescência das referências registradas nos artigos da SSCP no período de 2005 a 2016

Fonte: Dados da Pesquisa

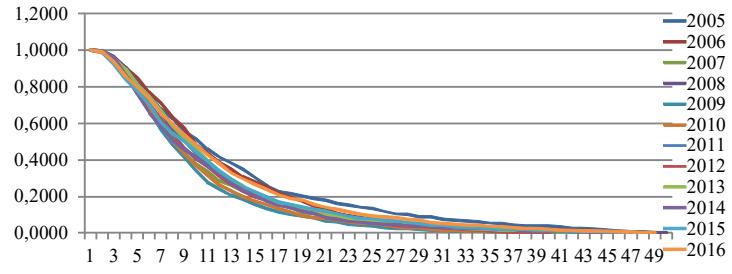

Figura 6 - Padrão de obsolescência das referências registradas nos artigos da SBM no período de 2005 a 2016

Fonte: Dados da Pesquisa

Nas Figuras 5 e 6, quanto mais próximas ao eixo $\mathrm{Y}$ as linhas descem, significa que, no ano correspondente, mais rápido se configurava o ritmo da perda da utilidade das informações presentes no referencial utilizado pelos autores. Ao passo que, quanto mais distante desse eixo ocorre a descida das linhas, mais lento é o processo de obsolescência da literatura observado naquele ano em questão.

De maneira geral, as linhas que representam a obsolescência da SSCP apresentam um padrão de perda de utilidade muito próximo em quase todos os anos analisados, pois, em 2009, esse processo se mostrava mais acentuado e, em 2015, a utilidade da informação indicava um processo de perda mais vagaroso.

A SBM também apresenta uma obsolescência da literatura semelhante entre os anos de 2005 e 2016 . Porém, apresenta maiores variações nos anos de 2005, no qual o ritmo de envelhecimento foi mais lento, e em 2009, quando a literatura apresentava um padrão de perda de utilidade mais acelerado.

Cabe ressaltar que as informações presentes nas Figuras 5 e 6 são coerentes com as vidas médias dos registros bibliográficos dos pesquisadores das duas subáreas analisados na Figura 3, no qual apresentam um perfil de consumo de literatura de tipo clássica-efêmera.

A Figura 7 demonstra a evolução da taxa de envelhecimento da literatura científica da SSCP e da SBM. Os valores indicados na Figura indicam o percentual de citações que a literatura deixa de receber a cada ano até se tornar obsoleta.

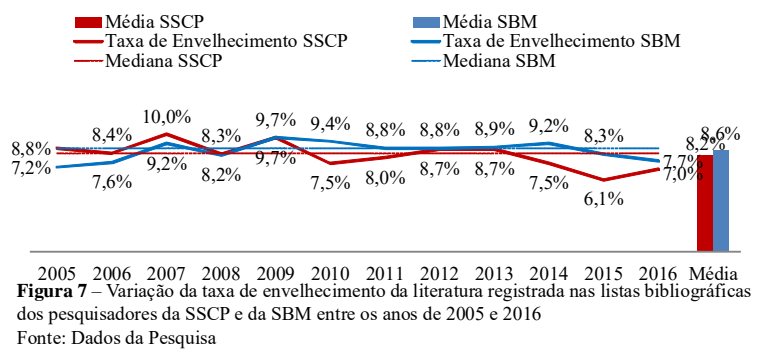

A Figura 7 demonstra que entre os anos de 2005 e 2007 a literatura utilizada na SSCP perdia utilidade em um ritmo mais rápido do que na SBM. Ao se comparar esse período com as 
informações sobre a tipologia de documentos utilizada em cada subárea (Tabela 3), esse panorama chama a atenção, uma vez que ocorreu no mesmo momento em que a SSCP reunia um maior quantitativo de livros e capítulos em suas listas bibliográficas, mas, mesmo assim, a subárea de tradição sociopedagógica apresentou uma taxa de obsolescência mais acelerada do que a de tradição biomédica.

Nos anos de 2008 e 2009, as taxas de envelhecimento observadas nas subáreas se igualam, ocorrendo uma inversão em 2010, quando a SBM passou a ter um ritmo de obsolescência mais acelerado que a SSCP. Ainda que ocorram oscilações nas taxas de envelhecimento nas e entre as subáreas, cabe ressaltar que esses indicadores apresentam um perfil semelhante em toda sua série histórica, conforme pode ser visto pelas médias ( $8,2 \% \mathrm{SSCP}$ e $8,6 \% \mathrm{SBM})$ e medianas $(8,4 \%$ SSCP e $8,8 \%$ SBM), onde as diferenças percentuais entre as subáreas são inferiores a $0,5 \%$ ano.

\section{Conclusões}

As análises das referências citadas, seus respectivos indicadores e comparações entre as subáreas da Educação Física no Brasil possibilitaram identificar e compreender algumas características da produção acadêmica do campo científico.

No que diz respeito ao tipo de documento utilizado, observou-se no período investigado que, na SBM, sempre houve uma ampla predominância no uso de artigos como base de sustentação argumentativa. Além disso, a tendência identificada é de crescimento no uso desse tipo de documento, em detrimento a outros formatos que, nessa subárea, têm apresentado uma tendência de extinção.

$\mathrm{Na}$ SSCP, o universo de referências identificadas demonstrou a existência de tipos de documentos mais diversificados. Nessa subárea, ainda que exista a predominância de artigos nas listas bibliográficas disponibilizadas pelos autores, os livros e capítulos de livro também aparecem como um formato importante para a sustentação teórico-argumentativa nos estudos sócio-pedagógicos.

Entretanto, existem evidências de que a política científica de avaliação adotada na área pode ter proporcionado uma alteração na cultura científica nessa subárea, na medida em que existe uma propensão de ampliação no uso de artigos de periódicos nas listas bibliográficas dos pesquisadores da SSCP, bem como uma tendência de redução no uso de outros tipos de literatura.

Sobre a vida média da literatura, foi identificado um perfil bastante semelhante nas duas subáreas, uma vez que, nos dois casos, a perda de utilidade da informação científica mostrou-se como clássico-efêmera ou intermediária, apresentando médias e medianas muito próximas no que se refere à taxa anual de obsolescência da literatura.

Por um lado, esse era o panorama esperado para a SBM em razão da sua histórica identificação com as áreas biomédicas, que demonstradamente, apresentam uma vida média do tipo intermediária. Por outro lado, porém, o achado é surpreendente, pois é senso comum na tradição epistemológica na área de Educação Física que a SSCP se aproxime das tradições científicas das Humanidades, em es- pecial, da área de Educação - fato que não se confirmou, indicando que as duas subáreas guardam muitas similaridades nas maneiras como utilizam e consomem a literatura científica que confere sustentação às pesquisas desenvolvidas na área.

Em suma, cabe ressaltar que as duas subáreas guardam mais similaridades do que diferenças no que diz respeito ao uso da literatura que confere sustentação teórica, metodológica e argumentativa à ciência praticada no campo científico de Educação Física.

\section{Referências}

Amadio,A., \& Barbanti, V.(2000). Biodinâmica do movimento humano e suas relações interdisciplinares. São Paulo: Estação Liberdade.

Araújo, C. A. (2006). Bibliometria: evolução histórica e questões atuais. Em Questão, 12(1), 11-32.

Betti, M., Carvalho, Y. M., Daolio, J., \& Pires, G. D. (2004). A avaliação da Educação Física em debate: implicações para a subárea pedagógica e sociocultural. Revista Brasileira de Pós-Graduação, 1(2), 183-194.

Bracht, V. (1993). Educação Física/Ciências do Esporte: que ciência é essa? Revista Brasileira de Ciências do Esporte, 14(3), 111-118.

Bracht, V. (2006). Por uma política científica para a educação física com ênfase na pós-graduação. In Fórum Nacional Permanente de Pós-Graduação Em Educação Física. Campinas, SP. Recuperado de http://www.cbce.org.br/ br/acontece/materia. asp?id=312

Bracht, V. (2007). Educação Física \& Ciência: cenas de um casamento (in)feliz. Ijuí: Unijuí.

Brookes, B. C. (1970). Obsolescence of special library periodicals: sampling errors and utility: sampling errors and utility contours. Journal of the American Society for Information Science, 21(5), 320-329.

Burton, R. E., \& Kleber, R. W. (1960). The «half-life» of some scientific and technical literatures. American Documentation, 11(1), 18-22.

Cardozo, J. M. C., Velasco, A. D., Domínguez, S. L., \& Reyes, J.A. M. (2019) ¿Qué se investiga en formación docente en educación física y en recreación? Retos, 36, 3-8. Recuperado de https://recyt.fecyt.es/index.php/retos/article/ view/51456

Carneiro, F. F. B., Ferreira Neto, A., \& Santos, W. dos. (2015). Práticas científicas em educação física: tradições e tensões. Curitiba: Editora CRV.

Carvalho, L. F. (2005). Bibliometria e saúde coletiva: análise dos periódicos Cadernos de Saúde Pública e Revista de Saúde Pública (Dissertação de Mestrado). Fundação Oswaldo Cruz, Rio de Janeiro.

Carvalho, L. F., Coimbra JR, C. E. A., Souza-Santos, R., \& Santos, R. V. (2007). Produção e citação em Saúde Coletiva: um olhar a partir dos periódicos Cadernos de Saúde Pública e Revista de Saúde Pública. Cadernos de Saúde Pública, 23(12), 3023-3030.

Carvalho, M. M. (1975). Análises bibliométricas da literatura de química no Brasil. Ciência da Informação, 4(2), 119141.

Carvalho, Y. M., \& Manoel, E. J. (2007). O livro como indica- 
dor intelectual na grande área da saúde. Revista Brasileira de Ciências do Esporte, 29(1), 61-73.

Carvalho, Y. M., Manoel, E. J., Novaes, B. C.A. C., Guirro, R. R. J., \& Bracht, V. (2008). O livro na pós-graduação: uma metodologia para avaliação do livro. Revista Brasileira de Pós-graduação, 5(10), 226-249.

Cassani, J. M., Santos, W., Carneiro, F. F. B., Oliveira, L. C. \& Ferreira Neto, A. (2020). Práticas Científicas em Circulação no GTT Memórias da Educação Física e Esporte do Colégio Brasileiro de Ciências do Esporte (2005-2017) Retos, 34, 295-299. Retos, 38, 472-481. Recuperado de https://recyt.fecyt.es/index.php/retos/article/view/75642

Centro de Gestão de Estudos Estratégicos. (2010). Doutores 2010: estudos da demografia da base técnico-científica brasileira. Brasília, DF: Centro de Gestão e Estudos Estratégicos.

Chacón-Borrego, F., Ubago-Jimenez, J. L., Garcia, J. J. G., Ruz, R. P. \& González, M. C. (2018). Educación e higiene postural en el ámbito de la Educación Física. Papel del maestro en la prevención de lesiones. Revisión sistemática. Retos, 34, 8-13. Recuperado de https://recyt.fecyt.es/ index.php/retos/article/view/54319

Coordenação de Aperfeiçoamento de Pessoa de Nível Superior. (2017). Relatório de Avaliação Educação Física: Avaliação Quadrienal 2017. Brasília, DF: Ministério da Educação.

Corder, S. M. (2004). Financiamento e incentivos ao sistema de ciência, tecnologia e inovação no Brasil: quadro atual e perspectivas (Tese de Doutorado). Universidade Estadual de Campinas, Campinas.

Daolio, J. (2007). O ser e o tempo da pesquisa sociocultural em Educação Física. Revista Brasileira de Ciências do Esporte, 29(1), 49-60.

Fensterseifer, P. E. (2001). A Educação Física na Crise da Modernidade. Ijuí: Unijuí.

Job, I. (2006). Análise bibliométrica das teses de uma comunidade científica em educação física com o uso do método indiciário. Revista Brasileira de Ciências do Esporte, 28(1), 201-216.

Kokubun, E. (2003). Pós-Graduação em Educação Física no Brasil: Indicadores objetivos dos desafios e das perspectivas. Revista Brasileira de Ciências do Esporte, 24(2), 9-26.

Larivière, V.,Archambault, E., Gingras, Y., \& Vignola-Gagné, E. (2006). The place of serials in referencing practices: comparing natural sciences and engineering with social sciences and humanities. Journal of the American Society for Information Science and Technology, 57(8), 997-1004.

Line, M. B., \& Sandison, A. (1974). An Obsolescence and changes in the use of literature with time. Journal of Documentation, 30(3), 283-350.

Manoel, E. J., \& Carvalho, Y. M. (2011). Pós-graduação na educação física brasileira: a atração (fatal) para a biodinâmica. Educação e Pesquisa: Revista de Educação da USP, 37(2), 389-406.

Maroldi, A. M., Lima, L. F. M., \& Hayashi, M. C. P. I. (2018). Vida Média e Obsolescência da Literatura em Educação Indígena. InCID: Revista de Ciência da Informação e Documentação, 9(1), 109-129.

Moya-Mata, I., Ros, C. Ros, Chacón, J. P. (2018) ¿Qué repre- sentan las portadas de los libros de texto de Educación Física en Primaria? Retos, 34, 295-299. Recuperado de https://recyt.fecyt.es/index.php/retos/article/view/63412

Nascimento, A. C. S. (2010). Mapeamento temático das teses defendidas nos Programas de Pós-Graduação em Educação Física no Brasil (1994-2008) (Tese de Doutorado). Universidade de São Paulo, São Paulo.

Noronha, D. P. (1998). Análise de Citações das dissertações e teses de doutorado em saúde pública (1990-1994): estudo exploratório. Ciência da Informação, 27(1), 6675.

Peña, M. P. \& Pedraz, M. V. (2018). Olimpismo en la revista Citius, Altius, Fortius (1959-1976): los inicios de la crítica al Movimiento Olímpico en España. Retos, 34, 177-182. Recuperado de https://recyt.fecyt.es/index.php/retos/ article/view/59959

Price, D. J. S. (1986). Little Science, Big Science... and beyond. Nova York: Columbia, University Press.

Rivero, A. C., Santos, R. N. M., \& Trzesniak, P. (2019). Políticas de avaliação e práticas de publicação nas Ciências da Saúde no Brasil. Revista Cubana de Información en Ciencias de la Salud, 30(1), 2-28.

Rodriguez, B. O., Carrasco-Legleu, C. E., Fierro, L. G L., CandiaLujan, K. F., Candia-Lujan, R. \& Longoria, R. J. N. (2019). Suplementos nutricionales en el tratamiento y la prevención del dolor muscular tardío: una revisión sistemática. Retos, 35, 407-412. Recuperado de https://recyt.fecyt.es/ index.php/retos/article/view/61908

Rodríguez, J. R., Rodríguez,A. S., \& Álvarez-Seoane, D. (2018). Revisión bibliográfica en el contexto español sobre investigaciones relacionadas con los libros de texto y materiales didácticos en educación física. Retos, 34, 363370. Recuperado de https://recyt.fecyt.es/index.php/retos/article/view/65902/39907

Saes, S. G. (2000). Estudo bibliométrico das publicações em economia da saúde, no Brasil, 1989-1998 (Dissertação de Mestrado). Universidade de São Paulo, São Paulo.

Souza, C. D. (2018). Impacto de las políticas brasileñas de ciencia y tecnología en la actividad investigadora de las universidades federales: un estudio cienciométrico del período 2003-2015 (Tese de Doutorado). Universidad Carlos III de Madrid, Madrid.

Strehl, L. (2005). O fator de impacto do ISI e a avaliação da produção científica: aspectos conceituais e metodológicos. Ciência da Informação, 34(1), 19-27.

Torres, T.Z. G., Magnanini, M. M. F., \& Luiz, R. R. Amostragem. (2009). In Medronho, R. A., Bloch, K. V., Luiz, R. R., \& Werneck, G. L. (Orgs.), Epidemiologia (pp. 403-414) São Paulo, SP: Atheneu.

Vanti, N. A. P. (2002). Da bibliometria à webometria: uma exploração conceitual dos mecanismos utilizados para medir o registro da informação e a difusão do conhecimento. Ciência da Informação, 31(2), 152-162.

Veloso, J. A Pós-graduação no Brasil: formação e trabalho de mestres e doutores no país (Vol. 2). Brasília: Fundação de Aperfeiçoamento de Pessoal de Nível Superior. 